\title{
A MULTI-CHANNEL CATHODE-RAY PHONOCARDIOGRAPH
}

BY

\author{
E. M. M. BESTERMAN AND J. K. HARRISON \\ From the Canadian Red Cross Memorial Hospital, Taplow, Bucks
}

Received November 2, 1952

The difficulties of recording the faint murmurs of early rheumatic valvulitis are well known, and for this reason it was considered advisable to develop a cathode-ray phonocardiograph. This is theoretically the ideal recording device owing to its minimal inertia and high sensitivity. This phonocardiograph is intended to provide objective confirmation of clinical findings on auscultation, recording change in murmurs during rheumatic activity and convalescence and at subsequent examinations. The patients, mainly children, are admitted to hospital shortly after the onset of rheumatic fever, and are not discharged until convalescence is completed.

The ear is more sensitive to sounds of high frequency than to those of low frequency, and although the actual intensity of the low-pitched heart sounds is far greater than that of high-frequency murmurs, the logarithmic low-frequency attenuation of the ear may cause them to sound equally loud. For this reason, filtering of the low-frequency sounds must be employed in order to record the murmurs satisfactorily. Mechanical filtering is employed in routine clinical auscultation by the use of the stethoscope bell or diaphragm. Rappaport and Sprague $(1941,1942)$ employ similar methods to record linear, stethoscopic, or logarithmic phonocardiograms. The first of these corresponds to the apex cardiogram without filtration, and the latter two filter the lower frequencies in increasing degree, so that the logarithmic record is equivalent to the auscultatory findings. In this country more stress has been laid on electrical methods of sound filtration (Leatham, 1949, and Cowen and Parnum, 1949), by attenuation of the lower and amplification of the higher frequencies. Various numbers of filters are employed by different workers, and we use four. The two low-pass filter positions are used for recording heart sounds and mitral diastolic murmurs, whereas the high-pass filter position (with maximal attenuation of low-pitch sounds) is best for recording aortic diastolic murmurs. The over-all gains on these amplifiers are $104 \mathrm{db}$. on the lowfrequency, and $101 \mathrm{db}$. on the high-frequency positions at $500 \mathrm{c}$./s.

\section{DESCRIPTION}

Microphone. A crystal microphone was selected owing to its high sensitivity, small size, and high signal-to-noise ratio. We use an Acos model (type MIC. 14-3) which has an almost flat response curve in the frequencies $30 \mathrm{c} . / \mathrm{s}$. to $1000 \mathrm{c}$. $/ \mathrm{s}$. The microphone is mounted in an ebonite holder which is attached to the chest by suction, the negative pressure being applied through a grooved lip, and not to the bell of the holder. An air-pressure equalizing valve maintains normal atmospheric pressure within the bell when this is applied to the chest and prevents damage to the diaphragm and crystal. This suction attachment of the microphone to the chest is preferable to the use of glue or straps, as it does not cause any noise between the case and the chest wall; and as the pressure of attachment is constant, comparable serial recordings are obtained. The microphones are connected to the amplifiers by coaxial cable.

The phonocardiograph amplifier (Fig. 1) consists of a low-microphone pentode followed by a double triode, the first section of which operates as an amplifier and the second as a phase splitter. The balanced output of the phase splitter feeds the output double triode, providing a balanced output voltage for the cathode-ray tube. Variable base-cut response control is provided by the switched cathode capacitors in the first valve and the coupling capacitors between first and second, and second and third stages. The heater 


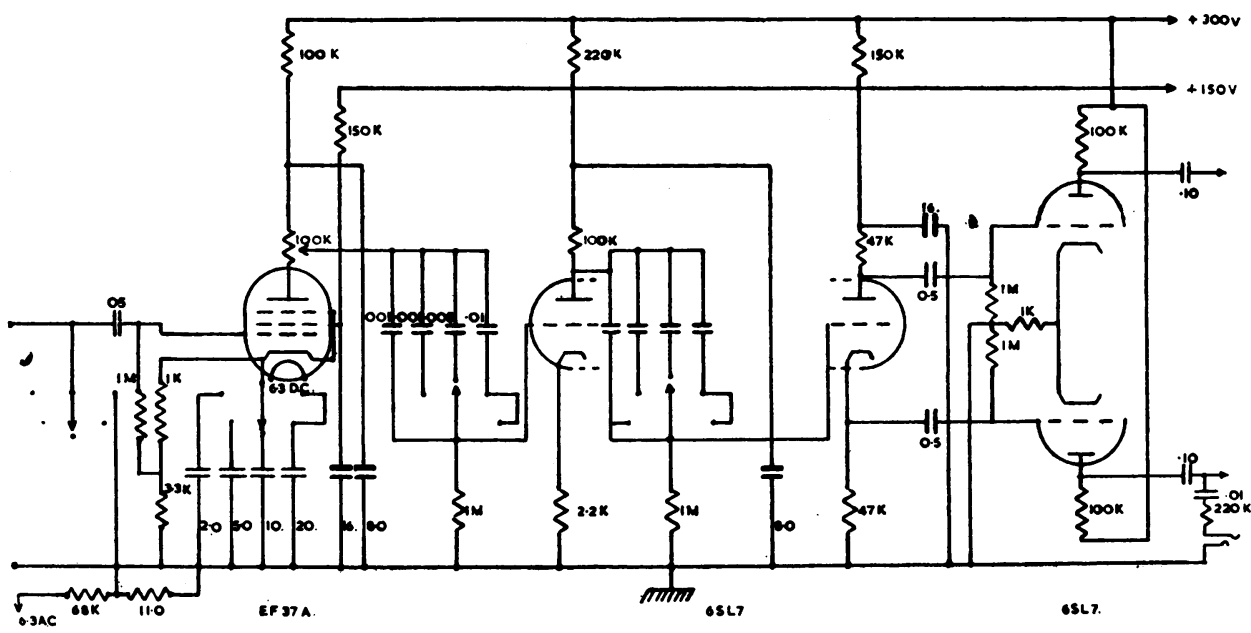

Fig. 1.-Phonocardiograph amplifier circuit.

of the first valve is fed from a D.C. supply, but those of the remaining valves are fed from A.C. Filtering is effected by low-frequency cut-off at different levels, four alternative filter positions being provided. Filtration of the higher frequencies is unnecessary owing to the low intensity of murmurs in this range, and the use of band-pass filters seems to offer little advantage in the analysis of murmurs which are complex noises and not pure sounds. The attenuation of these filters, relative to an input of $0.5 \mathrm{~m} . \mathrm{v}$. with $0 \mathrm{db}$. at $500 \mathrm{c}$. $/ \mathrm{s}$. is shown in Fig. 2 and compared with the human audiogram (after Fletcher, 1929). For calibration of amplification a 50-cycle signal may be introduced to the cathode-ray tube to produce a measurable deflection

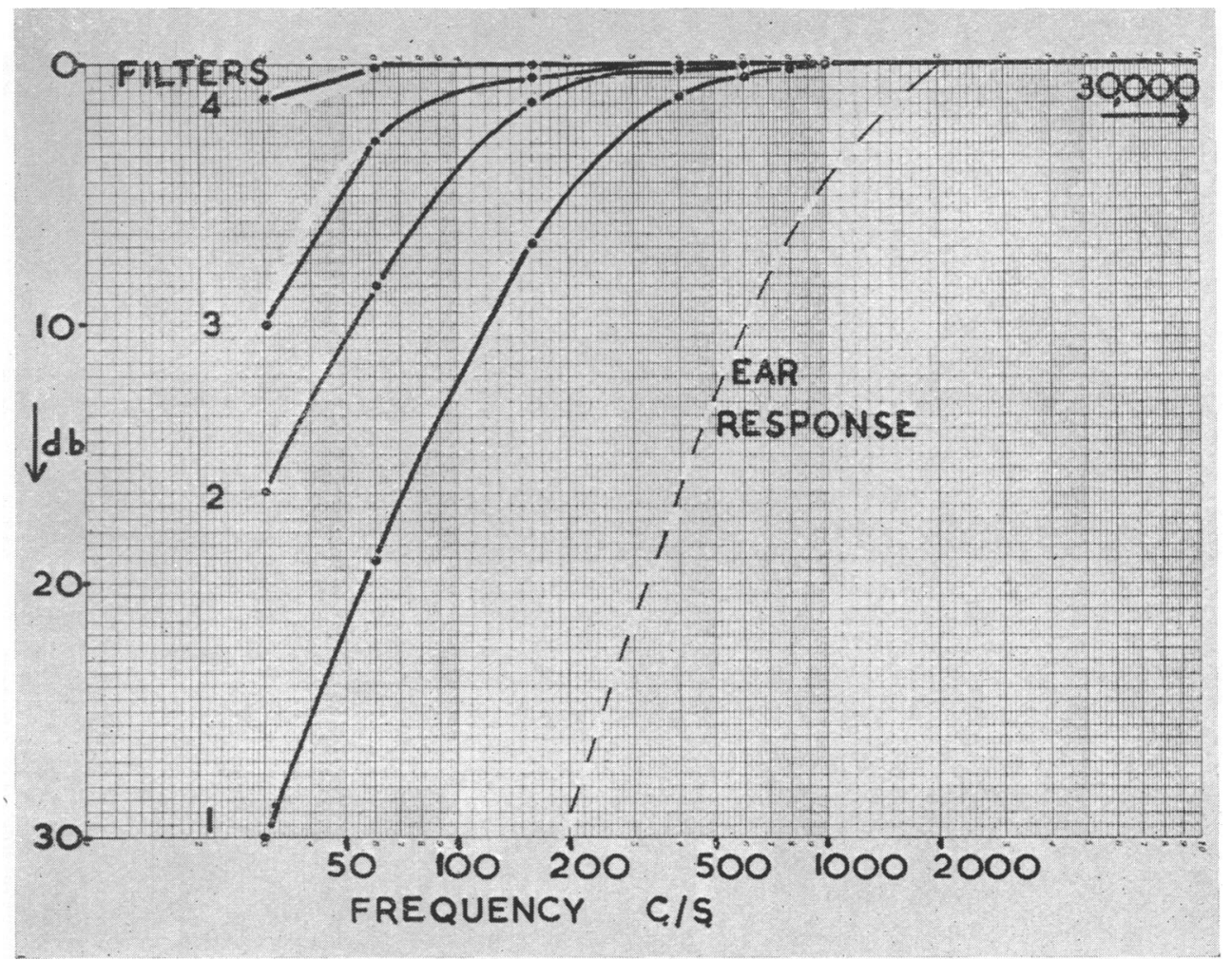

FIG. 2.-Frequency response curves of the four filter positions and of the human ear. 
at the same level of gain as that being employed for phonocardiography. Thus calibration of amplification is available, and is of value in assessing the changing intensity of murmurs over a period of time. Of course for this purpose the microphone must be applied at the same site on each occasion and the patient must be lying in the same position. Recordings are routinely made with the patient holding his breath in expiration. Provision for a headphone attachment is made in order that auditory " monitoring " may be employed.

A stabilized power supply provides H.T. for the amplifier. The cathode-ray tube is an electrostatic deflection tube with a blue screen of $1 \frac{3}{4}$ in. diameter, suitable for photography.

The electrocardiographic amplifier (Fig. 3) employs balanced stages, each consisting of a double triode valve. Long-tail-pair connections are used for the first two stages to provide coupling between the two halves and ensure a balanced output to the cathode-ray tube for balanced, unbalanced or differential input signals. The heaters of the valves are supplied as in the phonocardiograph amplifier. The electrocardiogram is routinely recorded by standard lead two. Alternatively, this amplifier may be used for recording the jugular phlebogram. A short rod of balsa-wood is connected to the diaphragm of a crystal micro-

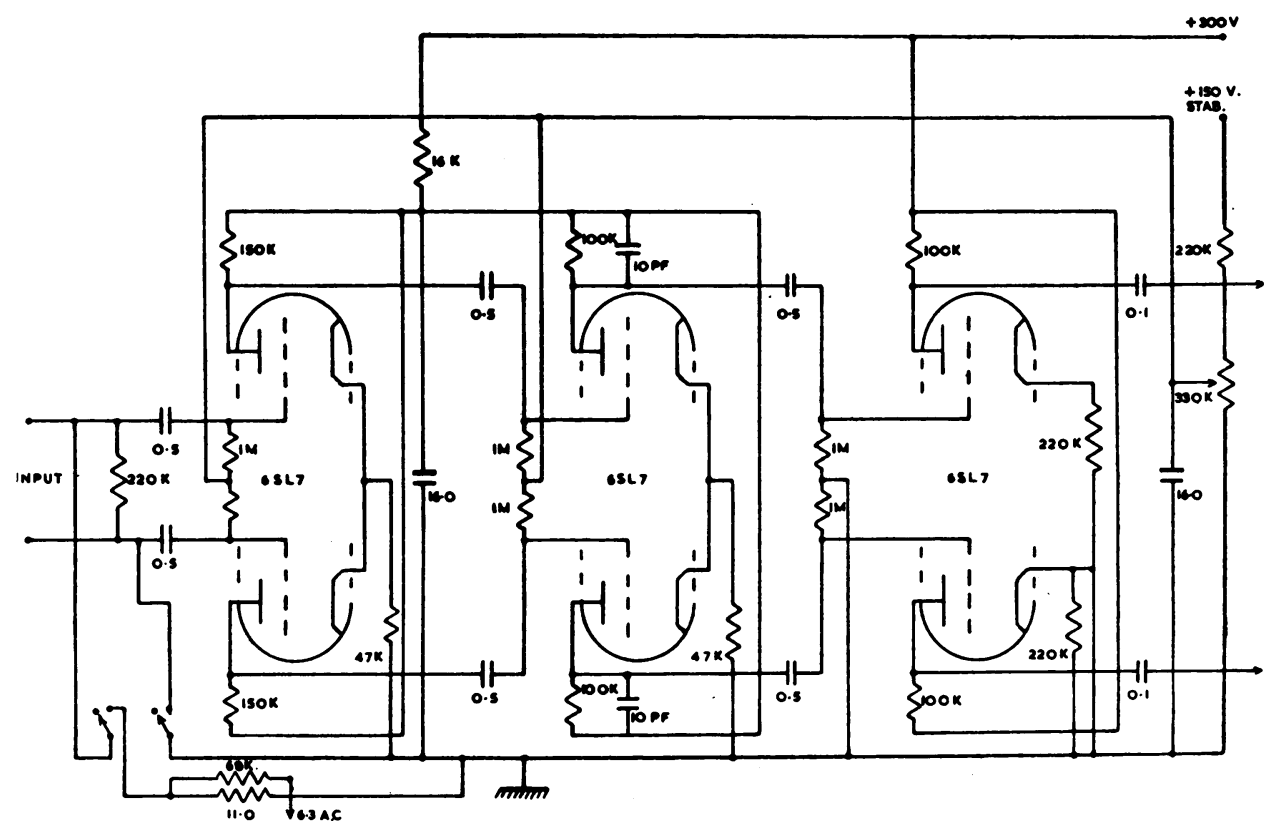

Fig. 3.-Electrocardiograph amplifier circuit.

phone and is used as the "pick-up" of the venous pulse. This microphone is then connected to the amplifier through the necessary resistance and capacitance to provide a suitable time-constant.

In the present machine four phonocardiograph and two electrocardiograph amplifiers are provided. In practice, however, it is only suitable for use on four channels, 3 phono-, 1 electro-cardiographic, which are quite adequate for routine use. These six amplifiers with C.R.O. tubes and the power unit form the lower part of the apparatus; the photographic unit being placed directly above.

The six cathode-ray tubes face forwards and the screens can be readily observed. Controls for brilliance and focus are provided. For recording, a hinged plane mirror is brought down before the cathode-ray tubes, forming an angle of approximately $45^{\circ}$ with the screens. This mirror reflects the cathode-ray images upwards and backwards to a concave mirror; from here the images converge on to the photographic paper. This paper is driven from the loading to the receiving cassette at a speed of $75 \mathrm{~mm}$./sec. Gearing is provided for altering this speed if required. A time marker records $1 / 10 \mathrm{sec}$. intervals at the edges of the paper. $100 \mathrm{ft}$. spools of Ilford recording papers, $120 \mathrm{~mm}$. width (B.P.I.) are used. The switch operating the motor drive simultaneously opens the shutter of the camera, making it impossible to operate the camera motor with the shutter closed. The complete equipment measures $36 \mathrm{in}$. high and $19 \mathrm{in}$. wide and is suitable for standard rack mounting.

\section{USE}

In practice two microphones are used simultaneously, one at the base and one at the apex. The basal murmurs are usually recorded at the high-pass filter position in order to accentuate the 
high-pitched aortic diastolic murmurs. The apical microphone is connected to two leads, so that if required, simultaneous apical records may be taken at high and low-pass filter positions on the remaining two phonocardiograph amplifiers. Thus high-frequency apical systolic and low-frequency mitral diastolic murmurs and heart sounds are recorded synchronously. The fourth amplifier records either electrocardiogram or phlebogram, as desired.

In order to reduce interference from outside noise a sound-proof room was constructed for these recordings. A small brick building, $14 \times 12 \times 11 \mathrm{ft}$. attached to the main building on only one wall was selected. The window was blocked and double doors inserted. Within this building an inner room, $6 \frac{1}{2} \times 8 \times 8 \mathrm{ft}$. was constructed, with a 6 in. concrete base, resting on coarse fibreglass which insulates it from the main structure. The walls are of brick and the roof of 6 in. concrete, one door being the only opening. The interior is lined with fibre-glass and perforated hard-board to prevent internal reflection of noise. Thus the inner room is isolated from all direct sources of noise conduction from the main building. The patient lies on a couch in this room, and the recording machine is in the outer room in order to eliminate transmission of noise from the camera motor to the microphones. A light signalling system provides communication between the attendant with the patient and the person operating the phonocardiograph. The outer room is used also as a dark room for immediate processing of the photographic paper.

In order to check the alignment of the traces on the four tubes, a common signal is injected, and any asynchrony is apparent when checking the photographic record with a set-square. A control on each amplifier allows adjustment of the X-shift to obtain correct alignment.

This machine has now been in routine use for one year, during which time numerous phonocardiograms have been obtained from 170 patients of all ages. As it is quite simple to expose many feet of paper during one recording, it is almost always possible to select a satisfactory tracing from even the most uncooperative patients. The principal difficulties arise from respiration and from chest muscle noises and in some cases recordings have to be taken during quiet respiration. Satisfactory photographic reproduction depends upon the brilliancy of the cathode-ray "spot" and on the extent and the frequency of its excursion. Thus a high-pitched, loud systolic murmur will appear less well defined than a mitral diastolic murmur of low-frequency and amplitude. In addition, satisfactory photographic development is essential if a good tracing is to be obtained. Examples of two phonocardiograms are shown in Fig. $4 \mathrm{~A}$ and B.

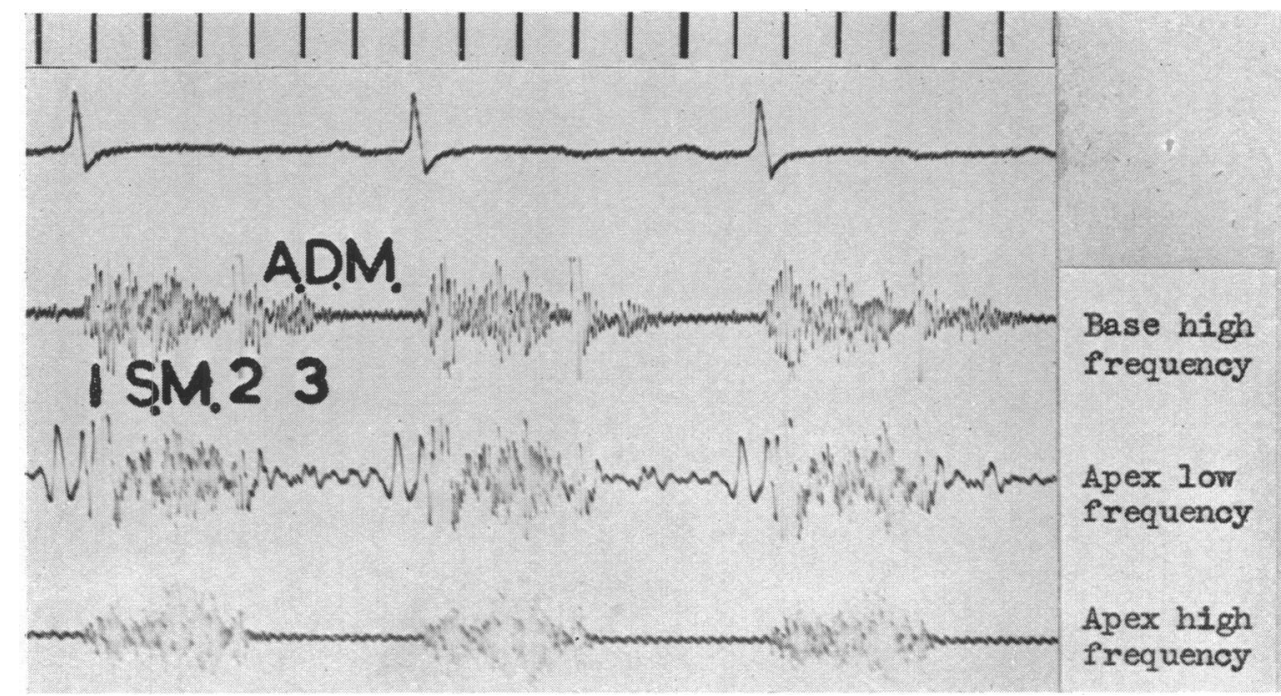

Fig. 4 A.-Systolic murmur and aortic diastolic murmur. 


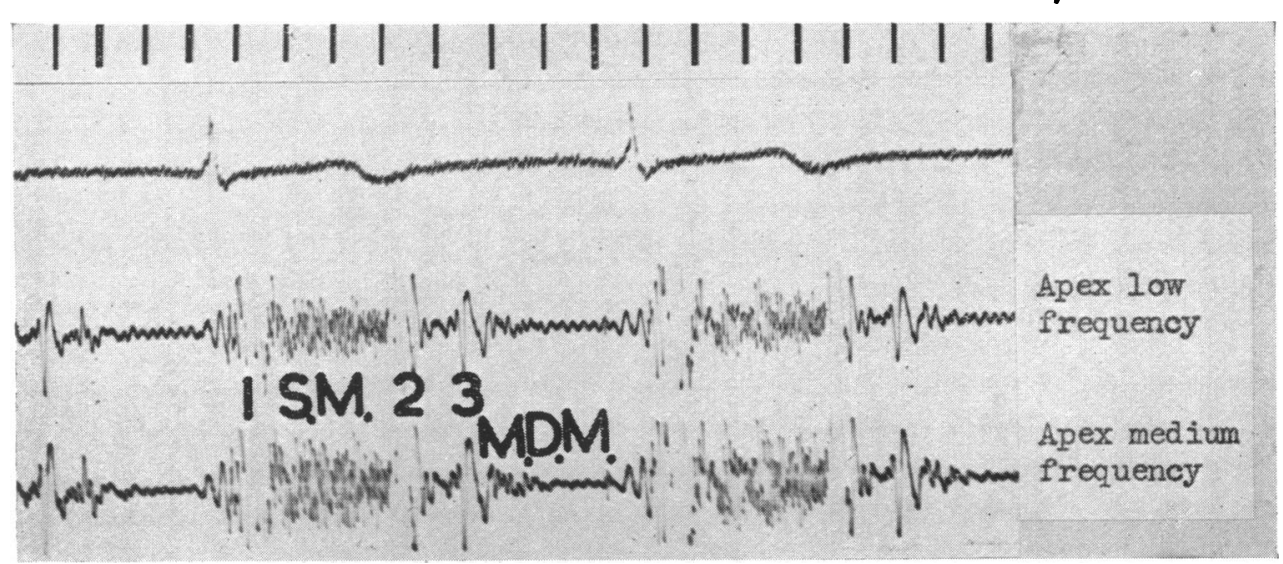

FIG. 4 B.-Mitral systolic and diastolic murmurs.

\section{SUMMARY}

A new multi-channel cathode-ray oscillograph phonocardiograph is described. This machine has been devised in order to study the murmurs of rheumatic carditis, and satisfactory recordings have been obtained of the faint diastolic murmurs frequently found in the early course of rheumatic activity.

This work was initiated at the suggestion of Dr. P. Wood, Dr. E. G. L. Bywaters, and Dr. G. T. Thomas, to whom we are extremely grateful. In addition, we are indebted to Mr. R. B. Archbold of the G.P.O. Research Station, Dr. L. Bernstein, and Dr. A. Leatham for much valuable technical advice. Dr. J. C. Simmonds and Dr. J. E. Varrall of Airmec Laboratories Ltd. undertook the final design and construction of this apparatus.

\section{REFERENCES}

Cowen, E. D. H., and Parnum, D. H. (1949). Brit. Heart J., 11, 356.

Fletcher, H. (1929). Speech and Hearing. New York.

Leatham, A. (1949). Postgrad. med. J., 25, 568.

Rappaport, E. E., and Sprague, H. B. (1941). Amer. Heart J., 21, 257.

- (1942). Amer. Heart J., 23, 59. 